\title{
Investigating the Presence of Mercury under a Dental Restoration Using Synchrotron K-Edge Subtraction Imaging
}

\author{
Assem Hedayat $^{1, *}$, George Belev ${ }^{2}$, Ning Zhu $^{3}$, Toby Bond ${ }^{3}$ and David Cooper ${ }^{4}$ \\ 1. College of Dentistry, University of Saskatchewan, Saskatoon, Canada. \\ 2. Saskatchewan Structural Sciences Centre, University of Saskatchewan, Saskatoon, Canada. \\ 3. Canadian Light Source, Saskatoon, Canada. \\ 4. Department of Anatomy and Cell Biology, University of Saskatchewan, Saskatoon, Canada. \\ * Corresponding author, assem.hedayat@usask.ca
}

Introduction: Mercury is a major constituent of dental amalgam. Patients with dental amalgam restorations intake mercury vapor by inhalation, may ingest elemental mercury, or may swallow tiny fragments of amalgam [1]. X-ray absorption near-edge spectroscopy (XANES) of extracted teeth that were restored with dental amalgam for more than 20 years identified dentinal tubules as another possible portal for patients' intake of mercury [2]. K-edge subtraction (KES) imaging is a state-of-the-art capability at the Biomedical Imaging and Therapy (BMIT) beamline at the Canadian Light Source (CLS). The technique is based on subtracting two sets of micro-CT images from each other, one above and one below the K-edge energy of mercury, which is the contrast element of interest in this research. The subtraction ameliorates the visibility of mercury-containing structures and annuls the visibility of mercury-free structures in the images [3].

Motivation: The aim of this research is to apply KES imaging to investigate the presence of mercury in a tooth exhibiting metallic-like features under a non-amalgam restoration when scanned and analyzed using synchrotron radiation micro-CT imaging technologies.

Methods: We used a Stylus ${ }^{\circledR}$ LED flashlight (Streamlight, Eagleville, PA, USA) to select five teeth restored with non-amalgam materials from the tooth bank at the college of dentistry, University of Saskatchewan, Saskatoon Canada. The material is referred to as non-amalgam because we do not know the history of the tooth, and the materials used to restore it. We scanned those teeth in addition to two more that were restored with dental amalgam at the BMIT 05ID-2 beamline at $50 \mathrm{keV}$ and with a 4.3 microns pixel size for other research purposes. We followed the same scanning procedure that we pursued in a previous study [4]. Reconstruction and 3D visualization of the images were carried out using Fiji (ImageJ) and Avizo 9.0 (FEI, Hillsboro, OR, USA), respectively. To test the effectiveness of the KES imaging technique, we ran a pilot test on a composite of hydroxyapatite and dental amalgam as follows: Permite regular set dental amalgam (SDI Inc., Itasca, IL, USA) was triturated, left to set, then fragmented using a vice. A number of small fragments were then glued to thin hydroxyapatite plates that are about $5 \mathrm{~mm}$ x $5 \mathrm{~mm}$ in cross-sectional area to make a composite mass. Since the k-edge of mercury is $83.102 \mathrm{keV}$, we scanned the composite twice, once above and once below that value and within $1 \mathrm{keV}$ from it following the same procedure referred to earlier [4]. We scanned the amalgam fragments / hydroxyapatite composite rather than amalgam in teeth because the latter exhibited bright streaks artifacts that compromised the fine details in the micro-CT images. The success of this pilot experiment encouraged us to pursue KES imaging on one of the teeth that was restored with non-amalgam because the image reconstruction following its scanning at $50 \mathrm{keV}$ revealed a dense thin layer of material that could have been dental amalgam remnants under the non-amalgam restoration. 
Results: Micro-CT images of teeth restored with amalgam were so bright with streaks that overwhelmed the underlying structure of the tooth itself due to the absorption of the X-rays. On the other hand, 3D visualization of one of the teeth restored with non-amalgam revealed a dense, metal-like layer under the restoration as shown in figure 1 (a). The figure also shows dense areas of dentin. These dense areas prompted us to investigate whether the metal-like structure is dental amalgam remnants under the restoration and if the dense dentin is attributed to the migration of mercury inside the tubules.
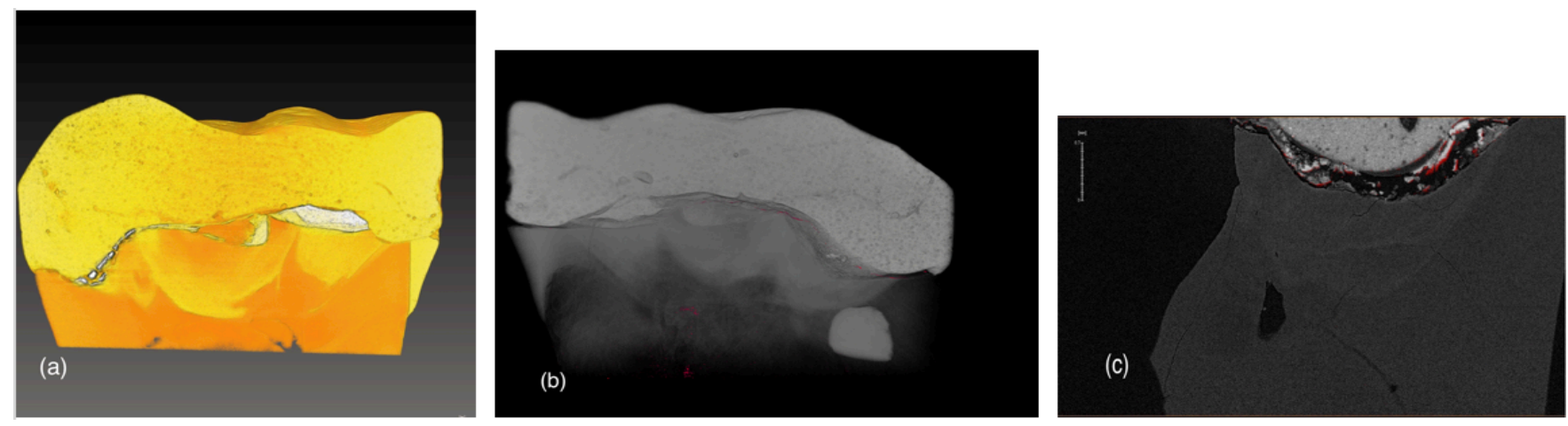

Figure 1. (a) 3D visualization of dense dentin under areas of metal-like structure (scanned at $50 \mathrm{keV}$ ) (b) KES image superimposed over the same image above the K-edge of mercury showing mismatch error in red (c) Mismatch error depicted in red.

Application of KES imaging of the same tooth in figure 1(a) investigated whether mercury is present in the dentinal tubules and the metal-like structures under the non-amalgam restoration. The mercury mapping indicated by the red color in figure 1(b) is indicative of a mismatch error due to cracks and other flaws in the tooth structure. This mismatch is elaborated in Figure 1(c) from another angle. If mercury is present, its concentration is below the detectable threshold of the KES experiment.

\section{Conclusions:}

1. KES imaging is a powerful tool to investigate the presence of mercury in dental structures.

2. KES imaging of the tooth in this study indicated the absence of mercury above the detection levels of KES under the non-amalgam restoration and in dentinal tubules.

3. Mismatch errors may indicate the presence of mercury when applying the KES imaging technique [5].

\section{References:}

[1] Scientific Committee on Emerging and Newly Identified Health Risks (SCENIHR). The safety of dental amalgam and alternative dental restoration materials for patients and users. 6 May 2008, p. 22. Retrieved from: http://ec.europa.eu/health/ph risk/committees/04 scenihr/docs/scenihr o 016.pdf

[2] H Harris et al, J. Synchrotron Rad. 15 (2008) 123

[3] Resourced from bmit.lightsource.ca

[4] A Hedayat et al, J. Synchrotron Rad. 23 (2016) 777

[5] We pursued the research described in this presentation at the BMIT facility at the CLS, which is supported by the Canada Foundation for Innovation, NSERC, the University of Saskatchewan, the Government of Saskatchewan, Western Economic Diversification Canada, the National Research Council Canada, and the CIHR. 